\title{
Big Data em Organizações de Médio e Grande Porte do Setor Público Brasileiro: \\ Prontidão e Situação Atual, Replicação do Estudo Holandês de Klievink et al. (2017)
}

\author{
Evandro O. Schaulet ${ }^{1}$, Guilherme Trez ${ }^{1}$ \\ ${ }^{1}$ Escola de Gestão e Negócios - Universidade do Vale do Rio dos Sinos (UNISINOS) \\ Av. Dr. Nilo Peçanha, 1600 - CEP 91330-002 - Porto Alegre - RS - Brazil \\ evandroschaulet@gmail.com, gtrez@unisinos.br
}

\begin{abstract}
Resumo. Este estudo é a replicação da pesquisa de Klievink et al. (2017) aplicada no governo holandês, utilizando as dimensões de alinhamento para tecnologia da informação, capacidades e maturidade organizacional. A questão de pesquisa é: em que medida as organizações do setor público brasileiro estão prontas e preparadas para utilização de iniciativas de big data? O resultado mostrou que os órgãos públicos brasileiros não estão prontos para a implementação de big data. As conclusões mostram que é necessário fomentar atividades na coleta de dados, compartilhando informações entre organizações e uma mudança de pensamento dos gestores públicos sobre a importância das informações na tomada de decisão.
\end{abstract}

\begin{abstract}
This study is a replication of the research by Klievink et al. (2017) applied in the Dutch government, using the alignment dimensions for information technology, capacities and organizational maturity. The research question is to what extent the Brazilian public sector organizations are ready and prepared to use big data initiatives. The result has shown that Brazilian public agencies are not ready for the implementation of big data. The conclusion pointed out that it would be necessary to encourage activities in data collection, sharing information among organizations and a change in the thinking of public managers about the importance of information in decision making.
\end{abstract}

\section{Introdução}

O setor público é responsável por utilizar os recursos provenientes dos impostos pagos pelos cidadãos da melhor maneira possível, devendo sempre buscar o melhor retorno para a sociedade [World Bank 2017]. No setor privado, a principal meta é alcançar o maior lucro possível através da produção e venda de produtos e serviços, gerando valor, mantendo uma vantagem competitiva e satisfazendo os clientes e partes interessadas de maneira sustentável [Kim et al. 2014]. O setor privado já utiliza big data como ferramenta de auxílio na gestão, principalmente para tomada de decisão e avaliação de mercados, concorrentes e consumidores. Já no setor público, mesmo havendo um crescimento no interesse sobre o potencial do big data, não se tem utilizado de forma efetiva e intensiva tecnologias de mineração de dados [Munné 2016]. 
Klievink et al. [Klievink et al. 2017] conduziram um estudo exploratório aplicado em organizações governamentais na Holanda, que trata da prontidão e das incertezas do governo em relação à implementação e utilização de big data, trazendo uma avaliação da disponibilidade de big data no setor público. O artigo dos autores avalia a disponibilidade de dados das organizações, examinando três componentes focais da estrutura: alinhamento organizacional para TI, maturidade organizacional e capacidade organizacional. Para avaliar o alinhamento organizacional para TI, as organizações públicas foram categorizadas com base em suas principais atribuições legais e atividades de dados, e também considerando os tipos de aplicações de big data em congruência.

O presente estudo pretende replicar a pesquisa anterior em um contexto diferente, para avaliar a prontidão para utilização de iniciativas de big data em órgãos públicos no ambiente brasileiro. Nesse cenário, tem-se a Holanda, um país desenvolvido e membro da União Europeia, e o Brasil, um país em desenvolvimento, que faz parte do BRICS, e com cultura e ambiente político completamente distintos, com diferentes tipos de organizações públicas. Também pretende-se investigar, por exemplo, se as descobertas do artigo de Klievink et al. [Klievink et al. 2017] são características apenas da Holanda ou podem se repetir no Brasil.

Diante das questões referentes à relevância do alinhamento, maturidade e capacidades organizacionais no que tange a preparação e organização do setor público para utilização de big data num contexto digital, tem-se o seguinte questionamento: em que medida as organizações do setor público brasileiro estão prontas e preparadas para utilização de iniciativas de big data? Este questionamento será respondido através da avaliação do alinhamento para TI, capacidade e maturidade organizacional em relação à utilização de big data no setor público brasileiro e do diagnóstico das fragilidades, lacunas e possíveis áreas de melhoria envolvidas no processo de implementação de big data no setor público.

\section{Alinhamento Organizacional para TI}

No modelo de alinhamento proposto por Henderson e Venkatraman [Henderson and Venkatraman 1993], o primeiro elemento é a estratégia de negócios, que no setor público se refere às atribuições legais do órgão. Com base nas entrevistas realizadas no estudo de Romijn [Romijn 2014], quatro tipos principais de atribuições legais foram escolhidas para selecionar os órgãos públicos: 1. Coordenação baseada em projetos 2 . Pesquisa, avaliação 3. Registro e documentação 4. Gestão e administração.

$\mathrm{O}$ segundo elemento se refere à infraestrutura e processos organizacionais. No estudo de Romijn [Romijn 2014], foram considerados como uso e coleta de dados. O terceiro elemento é a estratégia de TI, que é representada por três tipos de aplicações de big data, que são: a) avaliação de objeto/assunto; b) pesquisa; c) monitoramento contínuo.

O quarto elemento é a infraestrutura e processos de TI, e é formado por cinco características do big data: a) uso e combinação de vários conjuntos de dados, tanto de fontes de informações internas quanto externas [Davenport et al. 2012, Simon 2013]; b) uso e combinação de dados estruturados e não estruturados nas atividades de análise, através da combinação de dados de diversas fontes e formatos e técnicas de mineração de dados e algoritmos especializados [Adrian 2011]; c) grandes volumes de dados são 
recebidos, manipulados e analisados quase que em tempo real [Chen et al. 2014]; d) uso de algoritmos avançados, computação distribuída e tecnologia avançada necessários para execução de tarefas de computação de alta complexidade e volume [Simon 2013]; e) uso e fontes de dados existentes de maneira inovadora para aplicações novas e diferentes [Opentracker 2013].

O alinhamento entre a principal atribuição do órgão, juntamente com a análise do uso e coleta de dados, bem como das características e tipos de aplicação, permite que se encontre o alinhamento ideal, que é utilizado na avaliação dos órgãos através da aplicação do questionário, conforme Tabela 1.

Tabela 1. Alinhamento ideal

\begin{tabular}{|c|c|c|c|c|}
\hline Tipo de organização & $\mathbf{1}$ & $\mathbf{2}$ & $\mathbf{3}$ & $\mathbf{4}$ \\
\hline Principal atribuição legal & $\begin{array}{c}\text { Coordenação } \\
\text { baseada em } \\
\text { projetos }\end{array}$ & $\begin{array}{c}\text { Pesquisa, } \\
\text { avaliação }\end{array}$ & $\begin{array}{c}\text { Registro, } \\
\text { documentação }\end{array}$ & $\begin{array}{c}\text { Gestão e } \\
\text { Administração }\end{array}$ \\
\hline $\begin{array}{c}\text { Intensidade na atividade de } \\
\text { coleta de dados }\end{array}$ & Baixa & Baixa & Alta & Alta \\
\hline $\begin{array}{c}\text { Intensidade na atividade de } \\
\text { uso de dados }\end{array}$ & Baixa & Alta & Baixa & - em tempo real \\
\hline $\begin{array}{c}\text { Características mas } \\
\text { presentes no uso de big data }\end{array}$ & - & $\begin{array}{c}\text { conjunto de } \\
\text { dados Internos e } \\
\text { externos } \\
\text { - dados } \\
\text { estruturados e } \\
\text { não estruturados } \\
\text { - análise } \\
\text { complexa }\end{array}$ & $\begin{array}{c}\text { - conjunto de } \\
\text { externos } \\
\text { dados } \\
\text { - anólise } \\
\text { complexa }\end{array}$ & $\begin{array}{c}\text { - análise complexa } \\
\text { dados }\end{array}$ \\
\hline $\begin{array}{c}\text { Tipo de aplicação melhor } \\
\text { alinhada com big data }\end{array}$ & - & $\begin{array}{c}\text { Pesquisa } \\
\text { inovador de }\end{array}$ & $\begin{array}{c}\text { Avaliação de } \\
\text { objeto/assunto }\end{array}$ & $\begin{array}{c}\text { Monitoramento } \\
\text { contínuo }\end{array}$ \\
\hline
\end{tabular}

A Comissão Federal de Big Data dos EUA e Milakovich elencaram benefícios relevantes do uso de big data em vários setores para o governo dos EUA melhorar sua eficiência, eficácia e transparência, através de melhores informações, mais transparência e melhores insights sobre a realidade para suporte à tomada de decisão [Milakovich 2012]. Os três objetivos são transparência, eficácia e eficiência [Joseph and Johnson 2013, Weerakkody et al. 2011], que conectam os três tipos de aplicações de big data e seu valor agregado.

A presente pesquisa utilizou os elementos: alinhamento estratégico de TI e negócios, estratégia de negócios, identificação do nível do alinhamento organizacional através de pontuação.

\subsection{Capacidade Organizacional}

Na pesquisa de Klievink et al. [Klievink et al. 2017], os autores argumentam que, para alcançar os estágios do governo eletrônico, as organizações do setor público precisam de capacidades dinâmicas consideradas essenciais para o big data. Elas são: a) 
governança de TI [Zutshi and Sohal 2004], tomada de decisão e estruturas de responsabilidade, apoiando a organização [Valdes et al. 2011]; b) recursos de TI [Finney and Corbett 2007] e especialização para facilitar os atuais e novos sistemas de TI [Ngai et al. 2010]; c) atitude interna [Kamal 2006], principalmente abertura para tomada de decisão baseada em dados [Yeoh and Koronios 2010]; d) atitude externa [Daniel and Wilson 2003] e suporte para novos processos e sistemas com todos os principais envolvidos [Robey et al. 2008]; e) conformidade legal [Klievink and Janssen 2009], contemplando principalmente o uso de dados e proteção de privacidade, segurança e regulamentação da propriedade de dados [Ebrahim and Irani 2005]; f) gestão de dados [Lin and Hsia 2011. Wu and Hisa 2008], incluindo coleta, aquisição, controle de qualidade e parcerias [Wixom and Watson 2001]; g) experiência com ciência de dados [Wamba et al. 2017], especificamente, agregação de TI, core business e conhecimento de matemática [Schoenherr and Speier-Pero 2015].

Além disso, três dimensões dessas capacidades são utilizadas para avaliação: importância para o sucesso, possibilidade de desenvolver capacidade e presença atual. Nessa pesquisa, foram utilizados os seguintes elementos: estrutura e capacidades organizacionais, aprendizagem organizacional, capacidades dinâmicas, governo eletrônico, capacidade da infraestrutura e de processos.

\subsection{Maturidade Organizacional}

Este projeto se baseia no modelo de estágios de maturidade de Klievink e Janssen [Klievink and Janssen 2009], que auxilia no direcionamento da estrutura, inclusive com o desenvolvimento de capacidades, permitindo evoluir entre os estágios. O modelo dos autores tem como base cinco estágios de governo eletrônico: a) estágio 1 : stovepipe ${ }^{1} ; \mathrm{b}$ ) estágio 2: organizações integradas; c) estágio 3: portal nacional; d) estágio 4: integração interorganizacional; e) estágio 5: governo integrado e orientado por demandas.

Os cinco estágios de crescimento são apresentados em três aspectos distintos, com o objetivo de garantir que as pontuações informadas pelos entrevistados no questionário forneçam uma visão na qual a avaliação da maturidade organizacional possa ser baseada de forma coerente: 1) atividades e compartilhamento de informações; 2) instalações de TI; 3) sistemas de dados.

$\mathrm{Na}$ avaliação da prontidão de big data, a maturidade do governo eletrônico pode ser utilizada para representar a posição atual da organização no desenvolvimento em termos de transformação, que é relevante para o planejamento das ações para o desenvolvimento em direção ao big data [Klievink and Janssen 2009]. Os autores utilizam, também, em conjunto, outro aspecto para avaliar em qual estágio de crescimento do governo eletrônico as organizações públicas poderiam ser classificadas: um fluxo de desenvolvimento ou de tempo nos sistemas e dados dentro das organizações, gerado através de observações em entrevistas com profissionais de órgãos públicos.

Considera-se os seguintes sistemas de dados: 1. digitalização, que relaciona-se com o estágio 1: stovepipe. 2. Business Intelligence (BI) \& Data Management (DM), que refere-se ao estágio 2: organizações integradas. 3. BI 2.0 e ao DM 2.0 que faz referência ao estágio 3: portal nacional. 4. dados abertos, que se encaixa no estágio 4:

\footnotetext{
1 Uma organização stovepipe é chamada assim por possuir uma estrutura que restringe parcial ou totalmente o fluxo de informações, prejudicando ou até mesmo impedindo a comunicação entre os departamentos ou outras organizações; organizações com tendência ao isolamento.
} 
integração interorganizacional. 5. big data, que relaciona-se com o estágio 5: governo integrado e orientado por demandas.

Ao conectar os cinco estágios de crescimento do modelo de maturidade do governo eletrônico ao desenvolvimento dos sistemas de dados usados nas organizações públicas nas últimas décadas, aumenta-se a visibilidade do processo e a percepção por parte dos profissionais de TI.

No presente estudo, foram utilizados os seguintes elementos: perspectiva de crescimento, gerenciamento organizacional, governo eletrônico, nível de desenvolvimento das organizações em termos de colaboração, interação com outras organizações públicas e sua área de TI.

\subsection{Principais Conceitos sobre Big Data}

World Bank [World Bank 2014] descreve big data como um imenso volume de dados, estruturados ou não, que, devido ao seu tamanho, métodos tradicionais de software e banco de dados não têm capacidade de processar. O termo big data frequentemente é vinculado a volume de dados, principalmente pelo aumento exponencial da disponibilização de dados globais [Chen et al. 2014]. Contudo, não se pode levar em consideração apenas o volume de dados [Russom 2011], mas também as características velocidade e variedade: daí surge o termo 3 Vs. [Sagiroglu and Sinanc 2013]. Laney [Laney 2001] foi o precursor desse conceito, ao estudar o advento das atividades de $e$ commerce, e também conceituou volume como a criação e coleta de grandes massas de dados, vinculado ao aumento constante da escala dos mesmos. Ele descreve velocidade como a condução rápida da coleta e análise dos dados para aproveitar ao máximo o valor das informações. Já variedade foi descrita pelo autor como os diferentes e variados tipos de dados, estruturados ou não, incluindo áudio, vídeo, páginas de Internet, textos, além de dados tabulares e estruturados.

Com o surgimento do big data, a questão da qualidade dos dados teve sua relevância destacada. Neste contexto, além dos $3 \mathrm{Vs}$, para extrair valor e viabilizar a implantação de big data, há um quarto "V": a veracidade, que está tendo cada vez mais reconhecimento [Saha and Srivastava 2014]. Raghupathi, W. e Raghupathi, V. [Raghupathi, W. and Raghupathi, V. 2014] também traz o conceito de 4 Vs, acrescentando ao modelo anterior o elemento da veracidade, no qual a análise e os resultados são considerados verdadeiros e livres de erros. A veracidade refere-se diretamente a problemas de inconsistência e qualidade de dados [Saha and Srivastava 2014]. Segundo Kune et al. [Kune et al. 2015], big data prepara as informações que agregam valor em grandes volumes de dados, organizando e extraindo as mesmas de várias fontes e que sofrem constantes alterações.

\section{Método}

Esta pesquisa é de natureza mista, qualitativa e quantitativa, de caráter exploratório e descritivo, replicando o já existente estudo sobre big data de Klievink et al. [Klievink et al. 2017].

\subsection{Etapa Exploratória}

O tipo de replicação utilizado neste artigo é denominado extensão. Este artigo modificou a área geográfica e população pesquisada. Por se tratar de uma replicação, foi 
utilizado o mesmo questionário do artigo original. $\mathrm{O}$ artigo foi traduzido para português e validado na etapa de pré-teste. O questionário utilizado nesta pesquisa está disponível em bitbucket.org/csbc2021/wcge/downloads/A1.pdf.

\subsection{Etapa Descritiva}

Realizou-se o pré-teste do questionário em três órgãos públicos, sendo um governo estadual, um ministério público estadual e uma prefeitura. O questionário é formado por 41 questões, sendo 12 perguntas abertas, 18 de múltipla escolha com escala Likert de 7 pontos e 11 de múltipla escolha, sendo oito dicotômicas. Como amostra, foram pesquisadas organizações do setor público brasileiro, abrangendo prefeituras de médio ou grande porte e também institutos de pesquisa, empresas de economia mista responsáveis pelo processamento de dados, ministérios públicos e tribunais de justiça. Ao final do processo, alcançou-se aproximadamente 500 registros, conforme demonstrado na Tabela 2:

Tabela 2. Quantidades população por tipo de órgão

\begin{tabular}{|l|l|c|c|}
\hline \multicolumn{1}{|c|}{ Tipo de órgão } & \multicolumn{1}{|c|}{ População alvo } & $\begin{array}{c}\text { Órgãos } \\
\text { contatados }\end{array}$ & $\begin{array}{c}\text { Amostra } \\
\text { final }\end{array}$ \\
\hline Municípios & $\begin{array}{l}\text { Conforme censo do IBGE realizado em 2015, existem } \\
\text { em torno de 300 municípios com mais de 150 mil } \\
\text { habitantes }\end{array}$ & 140 & 60 \\
\hline $\begin{array}{l}\text { Unidades } \\
\text { federativas }\end{array}$ & 26 estados mais o Distrito Federal & 16 & 8 \\
\hline $\begin{array}{l}\text { Ministérios } \\
\text { públicos estaduais }\end{array}$ & 26 estados mais o Distrito Federal & 22 & 12 \\
\hline $\begin{array}{l}\text { Tribunais de } \\
\text { contas estaduais }\end{array}$ & 26 estados mais o Distrito Federal & 23 & 7 \\
\hline $\begin{array}{l}\text { Tribunais de } \\
\text { justiça estaduais }\end{array}$ & 26 estados mais o Distrito Federal & 19 \\
\hline $\begin{array}{l}\text { Órgãos públicos } \\
\text { federais e } \\
\text { estaduais em geral }\end{array}$ & $\begin{array}{l}\text { Agências nacionais de regulação, estatais, fundações, } \\
\text { institutos/centros de pesquisa e tecnologia: } \\
\text { aproximadamente 100 registros }\end{array}$ & 44 & 11 \\
\hline TOTAL & $\mathbf{5 0 4}$ & $\mathbf{2 6 4}$ & $\mathbf{1 0 1}$ \\
\hline
\end{tabular}

Este estudo foi feito utilizando a técnica de análise descritiva, baseada no estudo original, em que os autores criaram uma fórmula de classificação com pesos a partir da soma das variáveis, agrupamentos e enquadramento dentro dos elementos alinhamento para TI, capacidades e maturidade organizacional. Toda a explicação, regras de validação das respostas, e detalhamento das fórmulas está disponível em bitbucket.org/csbc2021/wcge/downloads/A2.pdf. A partir dessas notas das avaliações, chega-se à classificação da prontidão em big data. Aplicando as regras de avaliação nas respostas dos questionários, detalhadas em bitbucket.org/csbc2021/wcge/downloads/A3.pdf, com base no mesmo modelo de avaliação do artigo que serve de base nesta replicação, obtiveram-se as notas nos três aspectos de big data: alinhamento, maturidade e capacidade. 


\section{Resultados}

O questionário aplicado foi analisado com o objetivo de avaliar a prontidão dos órgãos públicos brasileiros para o big data, que é determinada através das notas alcançadas pelas respostas do questionário. A seguir, serão descritas as informações utilizadas na análise dos resultados.

Houve um total de 101 organizações com respostas válidas, conforme Tabela 3.

Tabela 3. Quantidade de registros por tipo de tarefa organizacional

\begin{tabular}{l|l|l}
\hline Tipo de tarefa organizacional & Quantidade & Percentual \\
\hline 1. Coordenação/tarefas baseadas em projetos & 3 & $3 \%$ \\
\hline 2. Avaliação e Pesquisa & 31 & $31 \%$ \\
\hline 4. Gestão e Administração & 67 & $66 \%$ \\
\hline TOTAL & 101 & $100 \%$ \\
\hline
\end{tabular}

\subsection{Análise e Discussão dos Resultados}

Cruzando as informações da intensidade dos dados com o tipo de organização e as características de uso dos dados, obtém-se a pontuação A. A média geral foi de 1.73 pontos. Os tipos de aplicação e os objetivos em relação ao big data, avaliados a partir de questões relativas às necessidades, constituem a pontuação B. A pontuação final geral foi de 1.95. Na Tabela 4 estão as notas por tipo de organização e a média geral.

Tabela 4. Avaliação de alinhamento para TI (pontuação A + B)

\begin{tabular}{l|r|r|r|r|r}
\hline \multicolumn{1}{|c|}{ Tipo de tarefa organizacional } & 2-muito baixo & 3-baixo & 4-médio & 5-alto & $\begin{array}{c}\text { 6-muito } \\
\text { alto }\end{array}$ \\
\hline 1-Coordenação/tarefas baseadas em projetos & $0,00 \%$ & $0,99 \%$ & $0,00 \%$ & $0,99 \%$ & $0,99 \%$ \\
\hline 2-Avaliação/Pesquisa & $2,97 \%$ & $5,94 \%$ & $11,88 \%$ & $4,95 \%$ & $4,95 \%$ \\
\hline 4-Gestão/Administração & $17,82 \%$ & $13,86 \%$ & $24,75 \%$ & $6,93 \%$ & $2,98 \%$ \\
\hline TOTAL & $\mathbf{2 0 , 7 9 \%}$ & $\mathbf{2 0 , 7 9 \%}$ & $\mathbf{3 6 , 6 3 \%}$ & $\mathbf{1 2 , 8 7 \%}$ & $\mathbf{8 , 9 2 \%}$ \\
\hline
\end{tabular}

Tem-se como resultado geral da avaliação de alinhamento organizacional para TI de 3.68, se enquadrando em médio, conforme faixas explicadas na Tabela 5. Quando a nota for somada com a nota da capacidade e maturidade organizacional, para a nota final, será reenquadrada em uma escala de 1 a 5 , e neste caso ela será 2.68 . Na Tabela 5, pela média geral, verificou-se que a nota 4 (média) predomina.

Tabela 5. Faixas de pontuação de alinhamento para TI

\begin{tabular}{|c|c|c|c|c|c|}
\hline $\begin{array}{c}\text { Pontuação } \\
\text { (= A + B: intervalo 2-6 pontos) }\end{array}$ & $\begin{array}{c}\text { Muito } \\
\text { baixo }\end{array}$ & Baixo & Médio & Alto & Muito Alto \\
\hline $\begin{array}{c}\text { Avaliação } \\
\text { de alinhamento para TI }\end{array}$ & 2 & 3 & $\mathbf{4}$ & 5 & 6 \\
\hline
\end{tabular}

\subsection{Avaliação de Capacidade Organizacional}

As notas têm como pontuação os níveis de 1 a 5 , conforme demonstrado na Tabela 6. A média das notas de todas as 101 respostas foi de 2.50 pontos, na pontuação de 1 a 5 , 
representando exatamente $50 \%$, ou seja, uma avaliação intermediária em termos percentuais.

Tabela 6. Faixas de níveis de capacidade organizacional

\begin{tabular}{|l|c|c|c|c|c|}
\hline Pontuação média (\%) & $0 \%-60 \%$ & $\mathbf{6 1 \% - 7 0 \% *}$ & $71 \%-80 \%$ & $81 \%-90 \%$ & $91 \%-100 \%$ \\
\hline Avaliação de alinhamento & Muito baixo & Baixo & Médio & Alto & Muito Alto \\
\hline
\end{tabular}

\subsection{Avaliação de Maturidade Organizacional}

As notas têm como pontuação os níveis de 1 a 5, conforme a Tabela 7. A média das notas de todas as 101 respostas foi de 2.59 pontos, o que significa uma nota baixa na escala das faixas de maturidade.

Tabela 7. Faixas de níveis de maturidade organizacional

\begin{tabular}{|l|l|l|l|l|l|}
\hline Nível de maturidade & 1 & $\mathbf{2}^{*}$ & 3 & 4 & 5 \\
\hline Avaliação de alinhamento & Muito baixo & Baixo & Médio & Alto & Muito Alto \\
\hline
\end{tabular}

A nota da maturidade é baseada em perguntas sobre compartilhamento de informações e recursos de TI. Em ambos os casos, com a ocorrência predominante de notas baixas, há um cenário que demanda um trabalho de fomentar tanto o compartilhamento de informações quanto a melhora dos recursos de TI, para então gerar um impacto nas notas mais altas, e, consequentemente, aumentar a média das notas de maturidade organizacional.

\subsection{Avaliação de Prontidão em Big Data}

Utilizando em conjunto as notas dos três elementos principais desta pesquisa (alinhamento para TI, capacidade e maturidade), obtém-se a nota completa da prontidão para big data de cada órgão público respondente. Para isso, deve-se somar as pontuações de 1 a 5 de cada elemento, representando desde a nota muito baixa até muito alta. Essa soma gera uma faixa entre 3 e 15 pontos, que após é enquadrada conforme opções contidas na Tabela 8.

Tabela 8. Faixas de pontuação da prontidão em big data

\begin{tabular}{|c|l|}
\hline Faixa & Avaliação da prontidão em big data \\
\hline $3-5$ & Pronto para orientação na utilização de big data \\
\hline $\mathbf{6}^{*} \mathbf{7}^{*}$ & Pesquisa na utilização de big data em relação a possibilidades e requisitos \\
\hline $8-9$ & Pronto para planejamento e projeto inicial de big data \\
\hline $10-11$ & Pronto para promover maior desenvolvimento de big data \\
\hline $12-13$ & Pronto para desenvolvimento detalhado e testes com big data \\
\hline $14-15$ & Pronto para implementação de big data \\
\hline
\end{tabular}

Analisando a Figura 1, é possível verificar a avaliação mais alta nos tipos de organização 1 e 2 . 


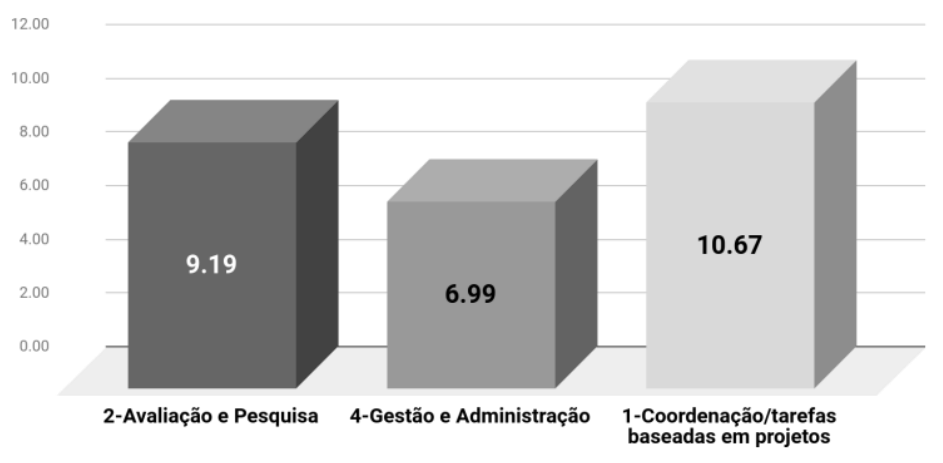

Figura 1. Média de avaliações por tipo de organização

Quanto à nota final, houve um resultado de 7.77 na escala da Tabela 9 , através da soma das notas dos três elementos base dessa pesquisa. A nota do alinhamento para TI aqui na avaliação final será ajustada para a escala 1 a 5 .

Tabela 9. Notas da avaliação final por elemento

\begin{tabular}{|l|l|l|}
\hline \multicolumn{1}{|c|}{ Elemento } & Faixa & Nota \\
\hline Alinhamento para TI & Médio & 2.68 \\
\hline Capacidade & Baixo & 2.50 \\
\hline Maturidade & Baixo & 2.59 \\
\hline NOTA FINAL & & 7.77 \\
\hline
\end{tabular}

\section{Considerações Finais}

Neste artigo buscou-se avaliar a prontidão dos órgãos públicos brasileiros para o big data, através da replicação do estudo holandês feito por Klievink et al. [Klievink et al. 2017]. O resultado da pesquisa mostra que os órgãos públicos brasileiros não estão prontos para implementação de big data, e que necessitam avançar muito mais na prontidão para o uso de big data. O estudo revelou que é necessário maior foco no fomento de atividades relativas à coleta de dados, principalmente através do compartilhamento de informações com outras organizações, tanto enviando quanto recebendo dados, gerando novas fontes de dados através do acesso a dados externos. Iniciativas de dados abertos auxiliam em muito neste item, pois possibilitam e facilitam a troca de informações entre órgãos públicos. Além disso, também evidenciou ser indispensável que ocorram melhorias da infraestrutura e instalações de TI, para possibilitar maior disponibilização e compartilhamento de aplicativos e dados com outras organizações. $O$ estudo também revelou que os órgãos públicos estão bem encaminhados quanto à geração e gestão dos dados pelos sistemas internos dos órgãos, que normalmente são softwares do tipo $\mathrm{GRP}^{2}$, que dão suporte às atividades administrativas.

A principal conclusão deste estudo remete a uma mudança de pensamento dos gestores públicos sobre a importância das informações na tomada de decisão. Isso é confirmado pela avaliação com relação à importância do apoio da alta gestão,

\footnotetext{
${ }^{2}$ Software de gestão pública. O mesmo conceito que ERP se refere a software para empresas, GRP é para governo
} 
fomentando ações que permitam a criação de um ambiente propício para governança de dados, com equipamentos e equipe trabalhando na área de ciência de dados, com acesso a parcerias e consultorias. Durante a pesquisa, identificou-se como fragilidades e lacunas em todas as organizações três pontos fundamentais: a questão da falta de apoio da alta gestão, a necessidade de acesso a consultorias e parcerias para auxiliar no processo de aprendizado em big data nas organizações e a carência e até inexistência de equipes multidisciplinares responsáveis pela atividade de ciência de dados.

Como contribuição prática, o entregável deste estudo se demonstra na Tabela 10, numa listagem de ações práticas sugeridas para auxiliar os órgãos públicos na evolução da prontidão do big data:

Tabela 10. Projetos estratégicos para evolução da prontidão do Big Data em órgãos públicos no Brasil

\begin{tabular}{|l|l|c|c|c|}
\hline \multirow{2}{*}{ ID } & \multicolumn{1}{|c|}{ PROJETO } & \multicolumn{2}{c|}{ ELEMENTOS } \\
\cline { 3 - 5 } & & $\mathbf{A}^{*}$ & $\mathbf{C}^{*}$ & $\mathbf{M}^{*}$ \\
\hline 1 & formação de equipe de cientistas de dados & $\mathrm{X}$ & $\mathrm{X}$ & \\
\hline 2 & compartilhamento/troca de informações entre os próprios órgãos públicos & $\mathrm{X}$ & & $\mathrm{X}$ \\
\hline 3 & apoio da alta gestão & & $\mathrm{X}$ & \\
\hline 4 & estimular a participação externa, com consultores e parcerias com outras organizações & & $\mathrm{X}$ & \\
\hline 5 & fomentar a utilização de ferramentas de BI nos órgãos de gestão e administração & & & $\mathrm{X}$ \\
\hline
\end{tabular}

* $\mathrm{A}=$ alinhamento $\mathrm{C}=$ capacidade $\mathrm{M}=$ maturidade

Como limitações, ocorreram principalmente dificuldades de acesso à base de informações, pois o contato com cada órgão público teve que ser realizado individualmente, assim como a busca pelo respondente dentro dos órgãos.

Como sugestão para futuros estudos pode-se citar a necessidade de continuidade da aplicação de replicações em outros cenários e contextos, para validar o framework do artigo original de Klievink et al. [Klievink et al. 2017], principalmente em outros países. Outra sugestão é estudar possíveis correlações entre os tipos de tarefa organizacional e tipos de órgão e as avaliações, para identificar características e fatores que possam determinar aspectos determinantes em relação à prontidão de big data, fornecendo informações que possam auxiliar outros órgãos públicos a se prepararem melhor para utilização de big data.

\section{References}

Adrian, M. (2011), Information Management Goes' Extreme': The Biggest Challenges for 21 st Century CIOs, SAS Campus Drive.

Chen, M., Mao, S. and Liu, Y. (2014). Big data: A survey. In Mobile Networks and Applications, pages 171-209.

Daniel, E. M. and Wilson, H. N. (2003). The role of dynamic capabilities in e-business transformation. In European Journal of Information Systems, pages 282-296.

Davenport, T. H., Barth, P. and Bean, R. (2012). How big data is different. In MIT Sloan Management Review, pages 22-24. 
Ebrahim, Z. and Irani, Z. (2005). E-government adoption: architecture and barriers. In Business Process Management Journal, pages 589-611.

Finney, S. and Corbett, M. (2007). ERP implementation: a compilation and analysis of critical success factors. In Business Process Management Journal, pages 329-347.

Henderson, J. C. and Venkatraman, H. (1993). Strategic alignment: Leveraging information technology for transforming organizations. In IBM Systems Journal, pages $472-484$.

Joseph, R. C. and Johnson, N. A. (2013). Big data and transformational government. In IT Professional, pages 43-48.

Kamal, M. M. (2006). IT innovation adoption in the government sector: identifying the critical success factors. In Journal of Enterprise Information Management, pages 192-222.

Kim, G-H., Trimi, S. and Chung, J-H. (2014). Big-data applications in the government sector. In Communications of the ACM, pages 78-85.

Klievink, B. and Janssen, M. (2009). Realizing joined-up government: dynamic capabilities and stage models for transformation. In Government Information Quarterly, pages 275-284.

Klievink, B., Romijn, B., Cunningham, S. and Bruijn, H. D. (2017). Big data in the public sector: Uncertainties and readiness. In Information Systems Frontiers, pages 267-283.

Kune, R., Konugurthi, P. K., Agarwal, A., Chillarige, R. R. and Buyya, R. (2015). The anatomy of big data computing. In Software: Practice and Experience, pages 79-105.

Laney, D. (2001) “3D Data Management: Controlling Data Volume, Velocity, and Variety. Application Delivery Strategies", blogs.gartner.com/douglaney/files/2012/01/ad949-3D-Data-Management-Controlling-Data-VolumeVelocity-and-Variety.pdf

Lin, L. M. and Hsia, T. L. (2011). Core capabilities for practitioners in achieving ebusiness innovation. In Computers in Human Behavior, pages 1884-1891.

Milakovich, M. (2012). "Anticipatory Government: Integrating Big Data for Smaller Government”. In: Internet, Politics, Policy 2012: Big Data, Big Challenges, Oxford.

Munné, R. (2016) "Big data in the public sector", New Horizons for a Data-Driven Economy: A Roadmap for Usage and Exploitation of Big Data in Europe, J. M. Cavanillas, E. Curry and W. Wahlster, New York, Springer, p. 195-208.

Ngai, E. W., Law, C. C. and Wat, F. K. (2008). Examining the critical success factors in the adoption of enterprise resource planning. In Computers in Industry, pages 584-564.

OpenTracker. (2013) "Definitions of big data", opentracker.net/article/definitions-bigdata, April.

Raghupathi, W. and Raghupathi, V. (2014). Big data analytics in healthcare: promise and potential. In Health Information Science and Systems, article 3. 
Robey, D., Im, G. and Wareham, J. D. (2008). Theoretical foundations of empirical research on interorganizational systems: assessing past contributions and guiding future directions. In Journal of the Association for Information Systems, article 4.

Romijn, J. H. (2014). Using big data in the public sector: Uncertainties and Readiness in the Dutch Public Executive Sector, Delft University of Technology.

Russom, P. (2011). Big data analytics, TDWI Research.

Sagiroglu, S. and Sinanc, D. (2013). Big data: A review. In 2013 International Conference on Collaboration Technologies and Systems (CTS), pages 42-47.

Saha, B. and Srivastava, D. (2014). Data quality: The other face of big data. In 2014 IEEE 30th International Conference on Data Engineering, pages 1294-1297.

Schoenherr, T. and Speier-Pero, C. (2015). Data science, predictive analytics, and big data in supply chain management: Current state and future potential. In Journal of Business Logistics, pages 120-132.

Simon, P. (2013). Too Big to Ignore: The Business Case for Big Data, John Wiley \& Sons Inc.

Valdes G., Solar M., Astudillo H., Iribarren M., Concha G. and Visconti M. (2011). Conception, development and implementation of an e-Government maturity model in public agencies. In Government Information Quarterly, pages 176-187.

Wamba, S. F., Gunasekaran, A., Akter, S., Ren, S. J. F., Dubey, R., and Childe, S. J. (2017). Big data analytics and firm performance: Effects of dynamic capabilities. In Journal of Business Research, pages 356-365.

Weerakkody, V., Janssen, M. and Dwivedi, Y. K. (2011). Transformational change and business process reengineering (BPR): Lessons from the British and Dutch public sector. In Government Information Quarterly, pages 320-328.

Wixom, B. H. and Watson, H. J. (2001). An empirical investigation of the factors affecting data warehousing success. In MIS Quarterly, pages 17-41.

World Bank. (2014) "Central America: Big Data in Action for Development", https://EconPapers.repec.org/RePEc:wbk:wboper:21325.

World Bank. (2017) Big Data in Action for Government: Big Data Innovation in Public Services, Policy, And Engagement: Solutions Brief", documents.worldbank.org/curated/en/176511491287380986/Big-data-in-action-forgovernment-big-data-innovation-in-public-services-policy-and-engagement.

$\mathrm{Wu}$, J. H. and Hisa, T. L. (2008). Developing e-business dynamic capabilities: an analysis of e-commerce innovation from I-, M-, to U-commerce. In Journal of Organizational Computing and Electronic Commerce, pages 95-111.

Yeoh, W. and Koronios, A. (2010). Critical success factors for business intelligence systems. In Journal of Computer Information Systems, pages 23-32.

Zutshi, A. and Sohal, A. (2004). A study of the environmental management system (EMS) adoption process within Australasian organisations-2. In Role of Stakeholders Technovation, pages 371-386. 